\title{
Ectasia dural e hipotensión endocraneal en síndrome de Marfán
}

\section{Dural ectasia and intracranial hypotension in Marfan syndrome}

\author{
Andrea Pichott ${ }^{\mathrm{a}}$, Tomás Bernstein ${ }^{\mathrm{b}}$, Guillermo Guzmán ${ }^{\mathrm{c}}$, \\ Guillermo Fariña $^{c}$, David Aguirred ${ }^{\mathrm{d}}$, Aníbal Espinoza ${ }^{\mathrm{e}}$
}

aRadióloga Pediátrica, Hospital San Juan de Dios, Santiago, Chile
${ }^{b}$ Neuroradiólogo, Hospital San Borja Arriaran, Santiago, Chile
`Neurólogo Infantil, Hospital San Borja Arriaran, Santiago, Chile
dNeurocirujano, Hospital San Borja Arriaran, Santiago, Chile
eRadiólogo Pediátrico, Hospital San Borja Arriaran, Santiago, Chile

Recibido: 23 de mayo de 2019; Aceptado: 01 de febrero de 2020

¿Qué se sabe del tema que trata este estudio?

La ectasia dural es un compromiso frecuente en síndrome de Marfán y otras conectivopatias, aunque poco conocido, que puede asociarse a síndrome de hipotensión endocraneana invalidante.
¿Qué aporta este estudio a lo ya conocido?

Presentar un caso de cefalea invalidante secundario a síndrome de hipotensión endocraneana, para advertir de esta complicación en niños portadores de conectivopatías, en especial síndrome de Marfán.

\section{Resumen}

Introducción: El síndrome de Marfán es un trastorno multisistémico del tejido conectivo de herencia autosómica dominante, de expresión variable. La ectasia dural es un compromiso frecuente, pero poco conocido, que puede asociarse a síndrome de hipotensión endocraneana (SHE). Objetivo: Presentar un caso de cefalea invalidante secundario a SHE, para advertir de esta rara complicación, que debe tenerse presente en niños portadores de conectivopatías, en especial síndrome de Marfán. Caso Clínico: Adolescente femenina de 13 años, portadora de sindrome de Marfán, de diagnóstico clínico según criterios de Ghent 2010, que consultó por cefalea ortostatica invalidante de 6 meses de evolución. La Resonancia Magnetica (RM) de cerebro mostró múltiples signos de hipotensión endocraneana, mientras que la RM de columna total mostró una ectasia dural que determinó la dilatación del saco tecal y remodelación posterior de los cuerpos vertebrales, especialmente a nivel del sacro. Se realizó tratamiento con parche sanguíneo autólogo epidural con buena respuesta clínica. Conclusiones: La ectasia dural, frecuente en el sindrome de Marfán, es una causa predisponente a fuga de líquido cefaloraquideo (LCR), que podría causar cefalea ortostática segundaria al SHE.
Palabras clave:

Marfán; ectasia dural; síndrome de hipotensión endocraneana; cefalea ortostática

Correspondencia:

Anibal Espinoza

anibalespinoza2005@yahoo.com 


\section{Abstract}

Introduction: Marfan syndrome is an autosomal dominant, multi-systemic connective tissue disorder of different presentations. Dural ectasia is a common, but little known complication that can be associated with intracranial hypotension syndrome (IHS). Objective: To present a case of severe headache secondary to IHS in order to warn about this rare complication, which must be considered in children carriers of connective tissue diseases, especially Marfan syndrome. Clinical Case: 13-yearold female carrier of Marfan syndrome, clinically diagnosed according to the 2010 Ghent criteria, who consulted due to a 6-months history of severe orthostatic headache. Head magnetic resonance imaging (MRI) showed multiple signs of intracranial hypotension, while whole-spine MRI showed dural ectasia that caused the thecal sac dilation and subsequent remodeling of vertebral bodies, especially the sacral ones. Treatment with an autologous epidural blood patch was administered with good clinical response. Conclusions: Dural ectasia, frequent in Marfan syndrome, is a predisposing cause of cerebrospinal fluid (CSF) leakage, which could cause orthostatic headache secondary to IHS.

\section{Keywords:}

Marfan;

dural ectasia;

intracranial

hypotension syndrome;

orthostatic headache

\section{Introducción}

El síndrome de Marfán es un trastorno multisistémico del tejido conectivo de herencia autosómica dominante, de expresión variable, aunque $25-30 \%$ de los casos son mutaciones esporádicas ${ }^{1,2}$. La incidencia estimada es $2-3$ en 10.000 nacidos vivos, sin predilección por género o grupo étnico ${ }^{1}$. Se han identificado mutaciones del gen de la fibrilina-1 (FBN-1) y de la señalización del factor de crecimiento transformante $\beta$ (TGF $\beta$ ), dentro de las alteraciones asociadas a este síndrome ${ }^{2}$.

El síndrome de Marfán puede afectar varios sistemas, incluyendo el cardiovascular, musculoesquelético, nervioso central, pulmonar, ocular y piel. En el diagnóstico se aplican los criterios de Ghent 2010 donde se valoran tres elementos diagnósticos principales: dilatación aortica $(Z$ score $\geq 2)$, ectopia lentis y estudio genético (FBN-1); los otros criterios mayores y menores definidos previamente en la versión de 1996 de Ghent, tienen hoy un valor menor y se agrupan como signos sistémicos, se les aplica una escala de valor (systemic score) que debe ser igual o mayor a 7 puntos, para ser significativos ${ }^{3,4}$.

La ectasia dural, definida como dilatación del saco dural y/o de las vainas de las raíces nerviosas, era considerada un criterio mayor en el diagnóstico del síndrome de Marfán (Ghent 1996), debido a su baja frecuencia en la población general y asociación a algunos trastornos específicos, tales como, síndrome de Marfán, síndrome de Ehlers-Danlos, neurofibromatosis, entre otros. Se presenta en $63-95 \%$ de los pacientes adultos con síndrome de Marfán y sobre el $40 \%$ de los niños con este síndrome ${ }^{5}$.

En la mayoría de los casos, la dilatación del saco dural ocurre en la región lumbo-sacra. Se plantea que la presión pulsátil continua del LCR, más alta a nivel lumbo-sacro con el paciente de pie, actuaría progre- sivamente en una pared dural y canal raquídeo debilitado y congénitamente defectuoso ${ }^{6}$. Esta condición de dilatación y adelgazamiento secundario del saco tecal y/o de las vainas de raíces nerviosas predisponen a fuga de LCR a través de pequeñas fistulas en la pared del saco tecal. La fuga de LCR es la única causa demostrada, ya sea iatrogénica como son las punciones lumbares o espontánea, para la ocurrencia de síndrome de hipotensión endocraneana (SHE) y sintomatología neurológica secundaria ${ }^{6}$. La ectasia dural y SHE son complicaciones poco conocidas del Síndrome de Marfán.

\section{Objetivo}

Presentar un caso de cefalea invalidante secundario a SHE, para advertir de esta rara complicación que debe tenerse presente en niños portadores de conectivopatías, en especial síndrome de Marfan.

\section{Caso Clínico}

Adolescente femenina, 13 años, consultó por cefalea de aparición progresiva de aproximadamente 6 meses de evolución. La cefalea se hacía evidente en forma súbita al ponerse de pie o sentarse, con intensidad de 10/10 y cedía a los 2 min de estar acostada, condición que limitaba severamente sus actividades de la vida diaria y la obligaba a permanecer en decúbito. Entre sus antecedentes familiares destacaba que su padre falleció a los 50 años debido a patología cardiovascular.

En etapa post menarquia (12 años), presentó un aumento de la talla puberal persistente, alcanzando en forma rápida una estatura de 1,83 mt. Además, refería dolores articulares.

En el examen físico destacó piel con abundantes estrías en región escápula, torácica y mamaria, paladar ojival, dientes apiñados, pectus excavatum leve simé- 
trico, soplo eyectivo en foco aórtico y escoliosis. En el examen de extremidades se apreciaba aracnodactilia, brazos largos y delgados, hiperlaxitud y signo de muñeca pulgar presente bilateral, limitación de la extensión en ambos codos logrando ángulo de $165^{\circ}$ y pie plano bilateral.

En la evaluación cardiovascular, el electrocardiograma fue normal y se pesquisó en ecocardiografía insuficiencia mitral mínima y leve dilatación de la raíz aórtica, con un índice $\mathrm{Z}$ score 3,5.

La radiografía de columna total antero-posterior y lateral objetivó incurvación de convexidad derecha de la columna en el plano coronal con ángulo de Cobb de $22^{\circ}$ e hipercifosis toraco-lumbar de $52^{\circ}$.

Las características descritas en el examen físico, sumado a los hallazgos en exámenes complementarios, plantearon el diagnostico de Síndrome de Marfan; aplicando criterios de Ghent 2010: la niña presenta dilatación de la raíz aortica ( $\mathrm{Z}$ score $\geq 2$ ), sumado a 7 o más puntos sistémicos (sistemic score) para el diagnóstico del síndrome.

Se realizó Resonancia Magnetica (RM) de cerebro y de columna, Tomografia Computada (TC) de columna lumbar. La RM de cerebro con contraste realizada para el estudio de su cefalea, mostró múltiples signos de hipotensión endocraneana (figuras 1 y 2) caracterizados por angulación caudal del tercio posterior del cuerpo calloso, descenso de las amígdalas cerebelosas, desplazamiento caudal del mesencéfalo sobre el puente, ingurgitación de plexos venosos e hipófisis y engro- samiento y realce dural supratentorial. Para buscar una causa de la hipotensión endocraneana se realizó una RM de columna total, que mostró una ectasia dural (figura 3) que determinó dilatación del saco tecal y re-

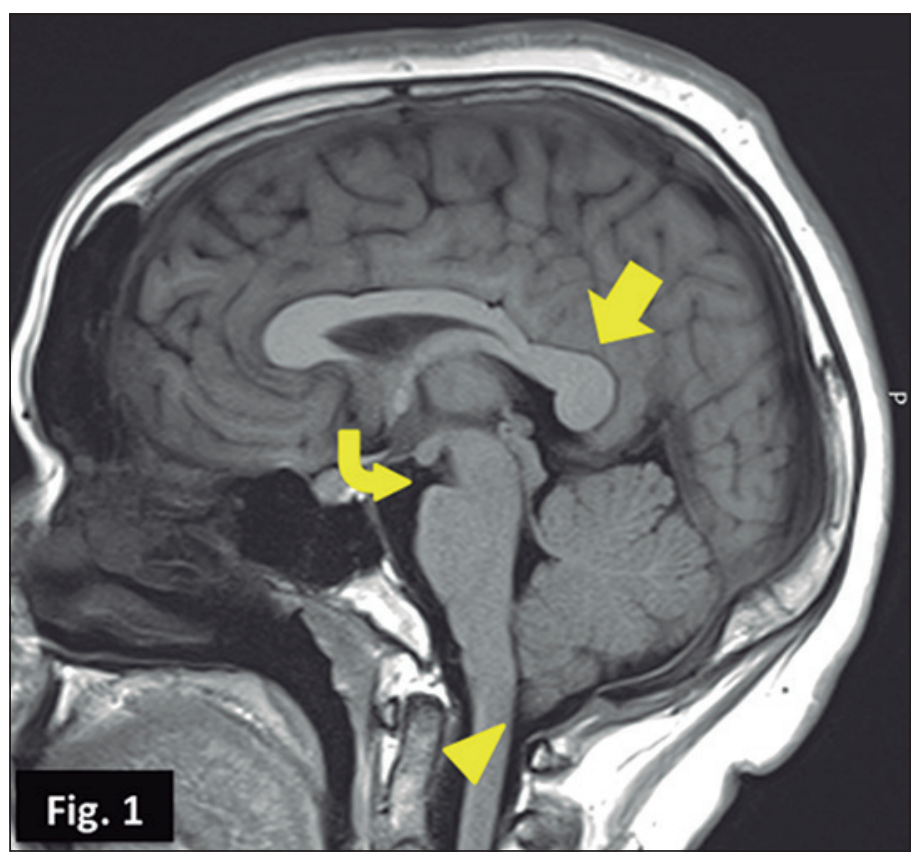

Figura 1. Resonancia Magnética (RM) de cerebro en secuencia T1 sagital sin contraste. Se reconocen signos de hipotensión endocraneana caracterizados por angulación caudal del tercio posterior del cuerpo calloso (flecha) y descenso de las amígdalas cerebelosas (cabeza de flecha). También se observa sutil desplazamiento caudal del mesencéfalo sobre el puente (flecha curva).
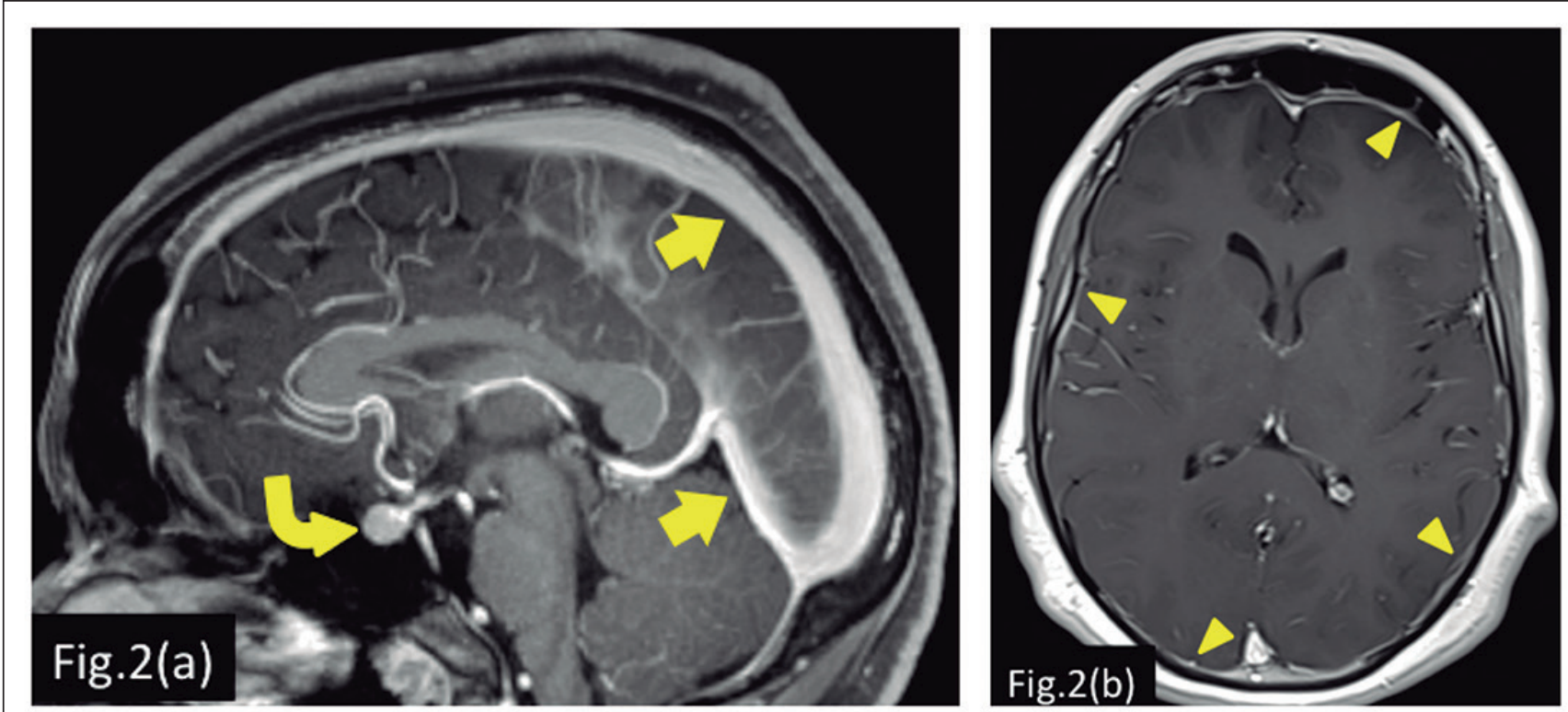

Figura 2. El uso de medio de contraste intravenoso es esencial para la caracterización de otros signos de hipotensión endocraneana visibles en Resonancia Magnética (RM) de cerebro. A. RM de cerebro en secuencia sagital T1 con contraste, evidencia ingurgitación de los senos venosos durales sagital superior y recto (felchas). También hay aumento de tamaño del glándula hipofisiaria (flecha curva). B. RM de cerebro en secuencia T1 axial con contraste, muestra engrosamiento y realce dural paquimeníngeo supratentorial bilateral simétrico (cabeza de flecha). 


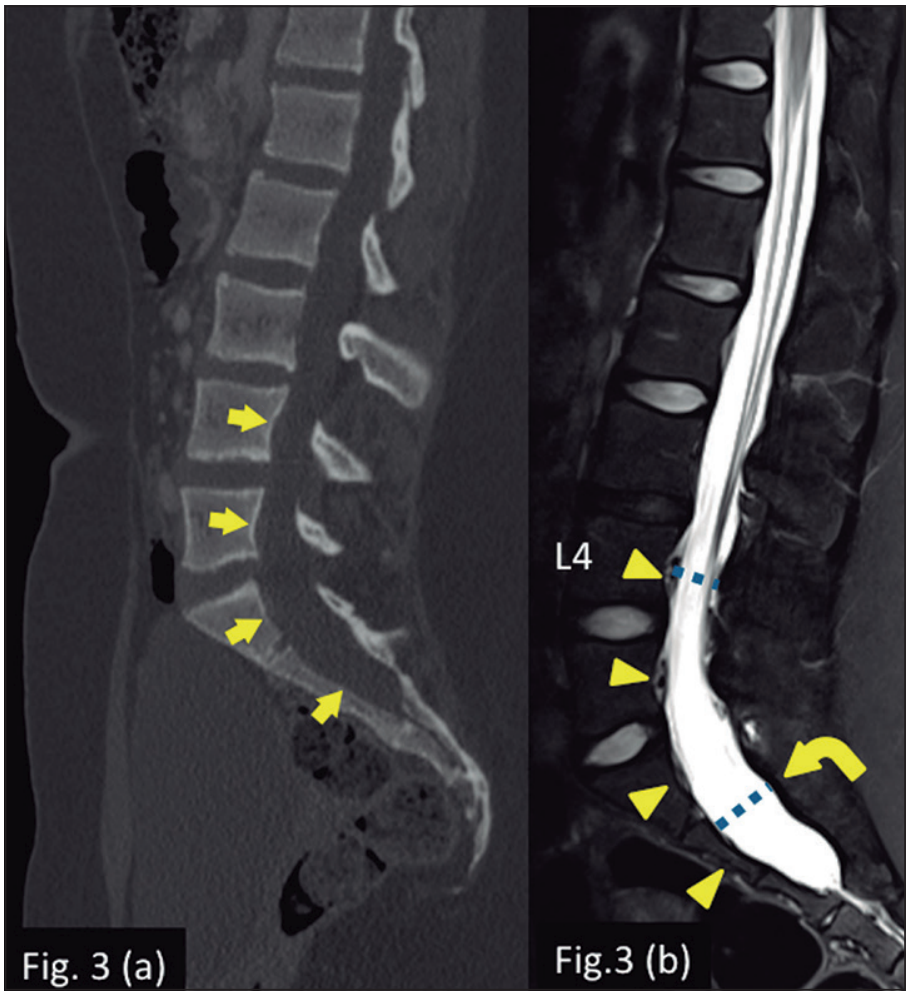

Figura 3. A. Reformateo sagital de tomografía computada (TC) de columna lumbar. Se observa remodelación de la pared posterior de los cuerpos vertebrales desde L4 a S3 (flechas). B. Resonancia Magnética (RM) de columna lumbar sagital potenciada en T2, confirma la presencia de una dilatación del saco tecal, que determina la remodelación posterior de los cuerpos vertebrales (cabezas de flecha). El ensanchamiento es más significativo a nivel del sacro (flecha curva), donde es más ancho que en L4 (líneas punteadas), criterio mayor de ectasia dural.

modelación posterior de los cuerpos vertebrales, lo que fue más significativo a nivel del sacro. La presencia de ectasia dural es predisponenete a fuga de LCR y SHE, no iatrogénico y seria la causa del cuadro de cefalea ortostática inavalidante de la niña.

Se realizo tratamiento con parche sanguíneo autólogo epidual, con escasa respuesta inicialmente $y$, en un segundo intento, se logró una buena respuesta terapéutica.

\section{Discusión}

La ectasia dural, en el síndrome de Marfán es generalmente asintomática, sin embargo, puede presentar síntomas neurológicos, tales como, lumbalgia, cefalea, dolor y parestesias en extremidades inferiores, dolor genital y rectal, que se exacerban al ponerse de pie ${ }^{7}$. Puede ir acompañada de espondilolistesis, escoliosis y meningocele sacro anterior, este último puede manifestarse como una masa pélvica.
La ectasia dural o dilatación del saco dural, con ampliación consecutiva del canal espinal, en cualquier segmento a lo largo de la columna vertebral, ocurre generalmente en las regiones lumbares y sacras inferiores, con adelgazamiento de la cortical de los pedículos y de las láminas de las vértebras, ensanchando los neuroforámenes, o la presencia de un meningocele anterior ${ }^{1,8}$.

En radiografía simple, se observa ensanchamiento de la distancia interpeduncular en la proyección anteroposterios y "scalloping" o festoneado del aspecto posterior de los cuerpos vertebrales en proyección lateral, con alta especificidad, pero baja sensibilidad ${ }^{9}$. La TC y RM han demostrado ser las modalidades de elección para el diagnóstico. Ofrecen una evaluación multiplanar y permiten una adecuada evaluación de la extensión de la dilatación del saco dural y/o de las vainas de las raíces nerviosas, presencia de meningocele o quistes aracnoidales asociados, y de las erosiones óseas en los aspectos posteriores de los cuerpos vertebrales, ensanchamiento de los neuroforámenes, en especial en la región lumbo-sacra ${ }^{1,8}$.

La RM tiene la ventaja de permitir una evaluación integral del neuroeje (medula/raíces y columna ósea), puede identificar la causa y evaluar diagnósticos diferenciales. La mielografía por tomografía computada (mieloTC) es un estudio donde a través de una punción lumbar se administra medio de contraste al espacio subaracnoideo y se realiza una adquisición por TC para buscar el sitio de filtración de LCR; este examen es altamente sensible y específico, sin embargo, utiliza radiación ionizante y es invasivo, por lo que se utiliza para casos que requieran un tratamiento particular ${ }^{10}$. Independiente del método, no es común lograr objetivar el sitio de la filtración ${ }^{11}$.

Ahn et al. describió en RM, que la ectasia dural está presente si existen un criterio mayor o dos menores. Criterios mayores: Ancho del saco dural por debajo de S1 mayor que en L4 o sobre L4; Presencia de un meningocele sacro anterior. Criterios menores: Festoneado en aspecto posterior del cuerpo vertebral $\mathrm{S} 1>3,5$ $\mathrm{mm}$; Diámetro de la vaina de la raíz nerviosa $>6,5 \mathrm{~mm}$ a nivel de $\mathrm{L} 5^{12}$.

Oosterhof et al, también describe criterios cuantitativos para ectasia dural en RM, en ellos se calcula la relación del diámetro del saco dural dividido por el ancho de los cuerpos vertebrales en el plano sagital medio en los niveles L1 a S1 o DSR (dural sac ratio), tienen que ser mayores que 0.64 en L1, 0.55 en L2, 0.47 en L3, 0.48 en L4, 0.48 en L5 y 0.57 en S1 en pacientes adultos ${ }^{13,14,15}$. En niños no hay criterios establecidos, a pesar de esto, al aplican los criterios descritos a nuestra paciente, resultan positivos para el diagnóstico de ectasia dural.

Los diagnósticos diferenciales de ectasia dural incluyen quistes aracnoideos congénitos y tumores in- 
trarraquideos que ensanchan el canal medular. Cuando la ectasia dural se extiende a la pelvis como un meningocele sacro anterior, puede simular una masa pélvica ${ }^{16}$.

Una de las complicaciones conocidas de la ectasia dural es el SHE. Este síndrome puede ser producido por múltiples etiologías, sin embargo, todas tienen una fisiopatología en común: la fuga de líquido cefalorraquídeo (LCR) en algún punto del sistema nervioso central (SNC), siendo lo más frecuente a nivel dorsolumbar. El SNC es un sistema cerrado donde la presión intracraneana (PIC) es mayor a la atmosférica y puede variar en contexto de una enfermedad o de manera fisiológica como cambios en la posición (supino versus bipedestación) así como con maniobras como valsalva; esto explica la cefalea postural u ortostática, donde hay aumento de la intensidad al sentarse y disminuye al acostarse ${ }^{10}$.

La pérdida constante y abundante de LCR genera una disminución de la PIC y eventual succión y descenso del encéfalo, como seria el caso presentado. En un inicio la pérdida de PIC se compensa a través de mecanismos hemodinámicos que pueden ser identificados a través de métodos imagenológicos y plantear de manera prospectiva el diagnóstico de este síndrome. Las alteraciones se van haciendo progresivamente evidentes en la medida que los mecanismos de compensación se ven superados. Inicialmente se observa ingurgitación del sistema venoso y glándula hipofisiaria y engrosamiento y realce dural paquimeníngeo. En la medida que la pérdida de LCR se acentúa, el cuerpo calloso y tronco encefálico comienzan progresivamente descender. Esto se ve representado por angulación caudal del tercio posterior del cuerpo calloso, descenso de las amígdalas cerebelosas y desplazamiento caudal del mesencéfalo sobre el puente. Otro mecanismo es la formación de colecciones subdurales supratentoriales bilaterales simétricas, con o sin hemorragia asociada; éstas pueden alcanzar volúmenes significativos, e incluso llevar a una errónea atribución de la sintomatología. Eventualmente el descenso tronco encefálico puede llegar a la compresión y herniación a través del foramen magno, con alta morbi-mortalidad ${ }^{17}$.

En nuestro paciente no existía una causa conocida de la filtración de LCR (ej. válvula derivativa) por lo que se realizó una evaluación de columna total para intentar identificarla, determinando la presencia de ectasia dural. En general, las causas más frecuentes son roturas espontáneas de quistes perirradiculares o divertículos meníngeos. Tanto en niños como adultos se presentarán clínicamente como cefalea ortostática, sin embargo, a diferencia de los adultos, hasta el 54\% de los niños con SHE puede tener una enfermedad del tejido conectivo asociada ${ }^{18}$. Es relevante reconocer los hallazgos imagenológicos para orientar el estudio y realizar una evaluación sistémica cuando no se tenga el antecedente. Por otro lado, es importante tener presente que un paciente con síndrome de Marfan u otra enfermedad del tejido conectivo puede presentarse con cefalea ortostática por hipotensión endocraneana, por lo que debe estudiarse ya que tiene riesgo de morbimortalidad ${ }^{19}$.

El manejo dependerá de la ubicación y tamaño de la brecha meníngea, en nuestro caso se utilizó el parche de sangre en forma exitosa y es el tratamiento más utilizado. Los parches de fibrina también son comúnmente utilizados, sin embargo, estos son dirigidos al sitio de mayor sospecha del sitio de la filtración (ej. divertículo de mayor tamaño), por lo que requiere una evaluación imagenológica previa. La cirugía se reserva generalmente a los casos refractarios ${ }^{10}$.

\section{Conclusión}

La ectasia dural es una causa de hipotensión endocraneana que puede explicar cefalea ortostatica en pacientes con síndrome de Marfan, por ello es importante conocer esta complicación con eventual morbimortalidad, las técnicas de estudio y los hallazgos radiológicos de dicha patología.

\section{Responsabilidades Éticas}

Protección de personas y animales: Los autores declaran que los procedimientos seguidos se conformaron a las normas éticas del comité de experimentación humana responsable y de acuerdo con la Asociación Médica Mundial y la Declaración de Helsinki.

Confidencialidad de los datos: Los autores declaran que han seguido los protocolos de su centro de trabajo sobre la publicación de datos de pacientes.

Derecho a la privacidad y consentimiento informado: Los autores han obtenido el consentimiento informado de los pacientes y/o sujetos referidos en el artículo. Este documento obra en poder del autor de correspondencia.

\section{Conflicto de intereses}

Los autores declaran no tener conflicto de intereses. 


\section{Referencias}

1. Ha HI, Seo JB, Lee SH, et al. Imaging of Marfan syndrome: Multisystemic Manifestations. RadioGraphics 2007; 27:989-1004.

2. Robinson PN, Godfrey M. The molecular genetics of Marfan syndrome and related microfibrillopathies. J Med Genet 2000;37:9-259.

3. De Paepe A, Devereux RB, Dietz HC, Hennekam RC, Pyeritz RE. Revised diagnostic criteria for the Marfan syndrome. Am J Med Genet 1996;62:41726.

4. Loeys BL, Dietz HC, Braverman AC, et al. The revised Ghent nosology for the Marfan síndrome. J Med Genet 2010;47(7):476-85.

5. Fattori R, ANienaber C, Descovich B, et al. Importance of dural ectasia in phenotypic assessment of Marfan's syndrome. Lancet 1999; 354:910-3.

6. Knirsch W, Kurtz C, Häffner N. Dural ectasia in children with Marfan syndrome: A prospective, multicenter, patient-control study. Am J Med Genet.2006;140A:775-81.

7. Foran JR, Pyeritz RE, Dietz HC,
Sponseller PD. Characterization of the symptoms associated with dural ectasia in the Marfan patient. Am J Med Genet A 2005;134:58-65.

8. Lundby R, Rand-Hendriksen S, Hald JK, et al. Dural Ectasia in Marfan Syndrome: A Case Control Study. Am J Neuroradiol 2009;30:1534-40.

9. Ahn NU, Nallamshetty L, Ahn UM, et al. Dural ectasia and conventional radiography in the Marfan lumbosacral spine. Skeletal Radiol 2001; 30:338-45.

10. Amrhein TJ, Kranz PG. Spontaneous Intracranial Hypotension. Radiol Clin North Am. 2019;57(2):439-51.

11. Chazen J, Talbott J, Lantos J, Dillon W. MR Myelography for Identification of Spinal CSF Leak in Spontaneous Intracranial Hypotension AJNR Am J Neuroradiol. 2014; 35(10):2007-12.

12. Ahn NU, Sponseller PD, Ahn UM, et al. Dural ectasia in the Marfan syndrome: MR and CT findings and criteria. Genet Med. 2000; 2(3):173-9.

13. Oosterhof T, Groenink M, Hulsmans FJ, et al. Quantitative Assessment of Dural Ectasia as a Marker for Marfan Syndrome. Radiology. 2001;220:514-8.

14. Halewa E, Boileau C, Jondeau G,
Desperramons J, Pelage JP. Marfan Disease: Imaging Features. European Congress of Radiology 2014.

15. Weigang E, Ghanem N, Chang XC, Richter H. Evaluation of three different measurement methods for dural ectasia in Marfan síndrome. Clin Radiol 2006;61(11):971-8.

16. Sahin N, Genc M, Kasap E, Solak A, Korkut B, Yilmaz E. Anterior Sacral Meningocele Masquerading as an Ovarian Cyst: A Rare Clinical Presentation Associated with Marfan Syndrome. Clin Pract. 2015;5(2):752.

17. Upadhyaya P, Ailani J. A Review of Spontaneous Intracranial Hypotension. Curr Neurol Neurosci Rep. 2019;19(5): 22.

18. Schievink WI, Maya MM, Louy C, Moser FG, Sloninsky L. Spontaneous Intracranial Hypotension in Childhood and Adolescence J Pediatri. 2013; 163(2):504510.e3.

19. Su C, Lan M, Chang Y, Lin W, Liu K. Clinical Features, Neuroimaging and Treatment of Spontaneous Intracranial Hypotension and Magnetic Resonance Imaging Evidence of Blind Epidural Blood Patch Eur Neurol. 2009;61(5):301-7. 